\title{
NSW Annual Vaccine-Preventable Disease Report, 2011
}

\author{
Alexander Rosewell $^{\mathrm{A}, \mathrm{B}}$, Paula J. Spokes ${ }^{\mathrm{A}}$ \\ and Robin E. Gilmour ${ }^{\mathrm{A}}$ \\ ${ }^{\mathrm{A}}$ Health Protection NSW \\ ${ }^{\mathrm{B} C o r r e s p o n d i n g ~ a u t h o r . E m a i l: ~ a r o s w @ d o h . h e a l t h . n s w . g o v . a u ~}$
}

\begin{abstract}
Aim: To describe the epidemiology of selected vaccine-preventable diseases in NSW for 2011. Methods: Data from the NSW Notifiable Conditions Information Management System were analysed by: local health district of residence, age, Aboriginality, vaccination status, and organism, where available. Risk factor and vaccination status data were collected by public health units for case-patients following notification under the NSW Public Health Act 1991*.

Results: Outbreaks of measles and pertussis were reported in 2011, associated with unimmunised groups for measles, and a variety of factors for pertussis. Notification rates for other selected vaccine-preventable diseases remained stable. Conclusion: Vaccine-preventable diseases are generally well controlled in NSW. However, pertussis remains an important public health issue. To prevent measles high population vaccination coverage, including vaccination in risk groups, is essential.
\end{abstract}

The objectives of vaccine-preventable disease surveillance are, at an individual level, to identify events that may require immediate public health control measures, and at a population level, to identify risk factors such as age and geographic location that inform better targeted immunisation efforts.

This report describes notification data for measles, pertussis, rubella, Haemophilus influenzae serotype b invasive infection, invasive meningococcal disease, mumps, tetanus, and invasive pneumococcal disease in New South Wales (NSW) in 2011 and provides comparison with recent trends.

\section{Methods}

The notification requirements for medical practitioners, hospital general managers and laboratories under the state's public health legislation have been previously described. ${ }^{1}$ On receipt of a notification, a public health unit surveillance officer determines whether or not the notification meets the definition of a case of vaccinepreventable disease according to national criteria, ${ }^{2}$ and if so enters data gathered on each notified case into the NSW Notifiable Conditions Information Management System (NCIMS). In this report, a person with an illness that meets the case definition is called a case-patient.

Data describing cases in NCIMS were extracted for selected vaccine-preventable diseases with a date of onset in 2011. Rates were calculated using Australian Bureau of Statistics population estimates and are presented as annual rates per 100000 total population or population in age groups. Risk factor and vaccination status data were collected for case-patients through public health unit follow-up with general practitioners (GPs) and other sources such as case-patient or carer reports. The incidence of cases were analysed by geographic area of residence.

\section{Results}

Haemophilus influenzae serotype b invasive infection In 2011, four cases of $H$. influenzae serotype b infection were notified; this was the lowest annual incidence within the last decade. Three case-patients were aged less than 1 year and one was a 2 -year old child; all were male. Of the three infants aged less than 1 year, one 10-month old and one 11-month old infant were fully vaccinated for age (3 doses), while one 9-month old infant was partially vaccinated for age ( 2 doses). The 2 -year old case-patient was fully vaccinated (4 doses). No case-patients were identified as being Aboriginal. All lived in regional NSW.

\section{Measles}

In 2011, 90 cases of measles were notified in NSW, compared to 26 in 2010 . The highest case notification rates were reported among children aged 0-4 years (21 cases, 4.4 per 100000 population), of whom three were too young to be vaccinated, and in young people aged 10-14 years (17 cases, 3.7 per 100000 population) (Table 1). Thirty-nine case-patients (43\%) were male. Ten case-patients were identified as Aboriginal people, 


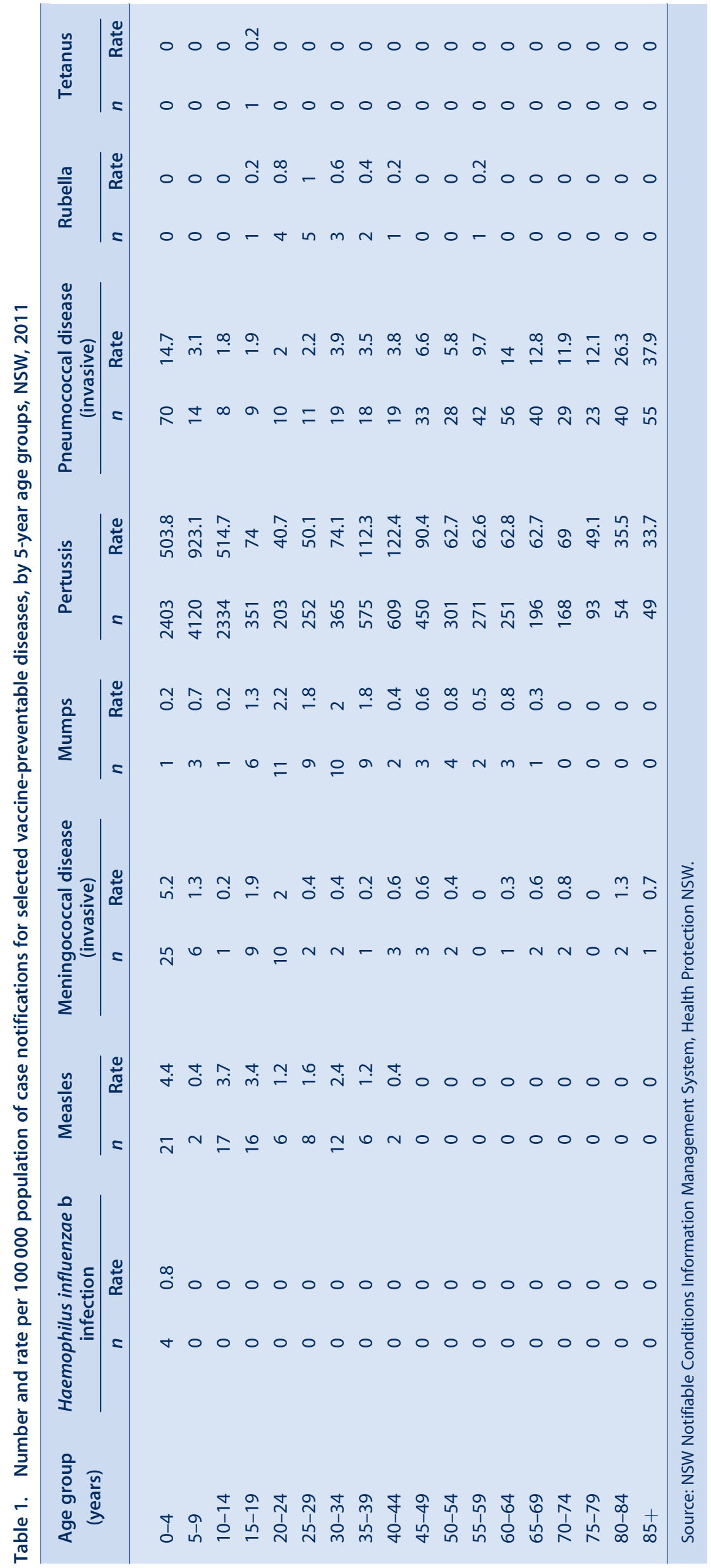




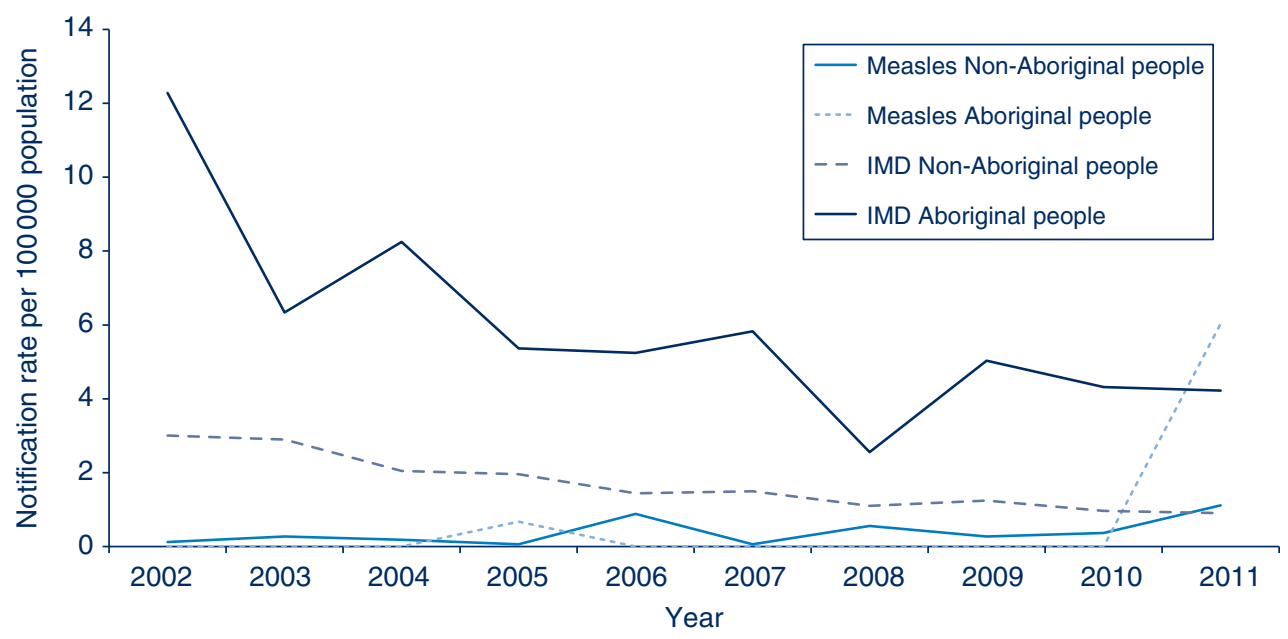

Figure 1. Case notifications of measles and invasive meningococcal disease (IMD) per 100000 population in NSW, for Aboriginal and non-Aboriginal people, for each year of the period 2002-2011.

Source: NSW Notifiable Conditions Information Management System, Health Protection NSW.

amongst whom case notification rates were significantly higher than in non-Aboriginal people (6.0 and 1.1 per 100000 , respectively) (Figure 1). Measles cases were notified in 10 local health districts (LHDs); the highest rate was in the Greater Southern LHD (10 cases, 4.9 per 100000 population) (Table 2).

Of the 90 cases, 50 (56\%) were unvaccinated, $18(20 \%)$ were vaccinated, and 22 (24\%) had missing vaccination status. Of the 18 vaccinated cases, five (all aged 15-35 years) had two documented doses, three one dose only and 10 did not have information on the number of doses of vaccine that they had received. Of the five fully vaccinated case-patients, vaccination history was validated by written records in three (one through the Australian Childhood Immunisation Register and two their personal health record), and for two based on self-report only. None of the nine case-patients who acquired their infection overseas had documented evidence of measles vaccination.

During the past decade, measles has been rare in NSW compared with the period before the 1998 Measles Control Campaign, with outbreaks primarily occurring in underimmunised populations and international travellers. Of the 90 cases notified in 2011, 12 (13\%) were acquired overseas, $11(12 \%)$ were epidemiologically linked to these cases, while in 67 (74\%) no link to an overseas-acquired case could be established. Among clusters in metropolitan Sydney, repeat presentations of case-patients to health settings prior to diagnosis were common. Source casepatients were retrospectively identified in emergency departments in several clusters. In two clusters, casepatients were children overdue for immunisation. ${ }^{3,4}$

In Western Sydney LHD, 26 cases (23 locally acquired) were notified from one local government area. Twelve of these were children from Pacific Islander communities, seven of whom attended the same high school. In Southern NSW LHD there were seven cases notified in an outbreak centred on a high school with low vaccination coverage in the Australian Capital Territory.

Internationally, there are eight different clades and 24 subclades of measles viruses referred to as genotypes. ${ }^{5}$ In 2011, 34 (38\%) cases had measles genotyping results. Of these, five (15\%) were measles genotype D8, seven (21\%) were measles genotype D4 and 22 (65\%) were measles genotype D9. Travel to France and Italy was associated with measles genotype D4 while travel to the Philippines was associated with measles genotype D9. In recent years, the measles virus D9 genotype has been identified in Europe and as endemic in selected countries of the AsiaPacific region, while measles virus D4 and D8 genotypes have been identified more widely. ${ }^{1}$

\section{Meningococcal disease (invasive)}

In 2011, 72 cases of invasive meningococcal disease were notified in NSW (62 confirmed and 10 probable), compared with 76 cases notified in 2010. Four deaths among case-patients were notified in 2011 across a wide age range, including: one infant aged less than 1 year, one 45-49-year old, one 70-75-year old and one 80-85-year old (all caused by serogroup B). This compares to five deaths in 2010 (three caused by serogroup B, one serogroup W135, and one with an unknown serogroup).

The highest case notification rates of invasive meningococcal disease were among children aged less than 5 years at onset of illness (25 cases, 5.2 per 100000 population) and young people aged 20-24 years (10 cases, 2.0 per 100000 population) (Figure 2). Of the case notifications among children aged less than 5 years, the highest rates 


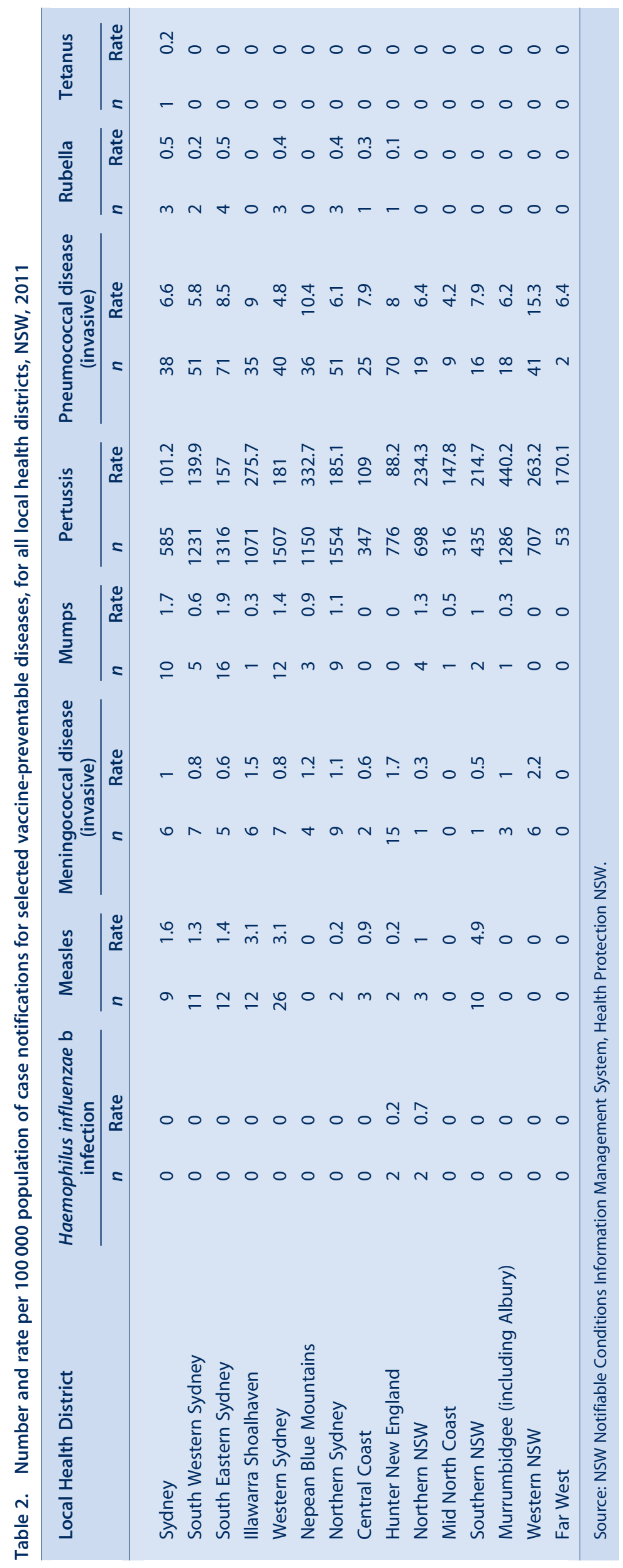




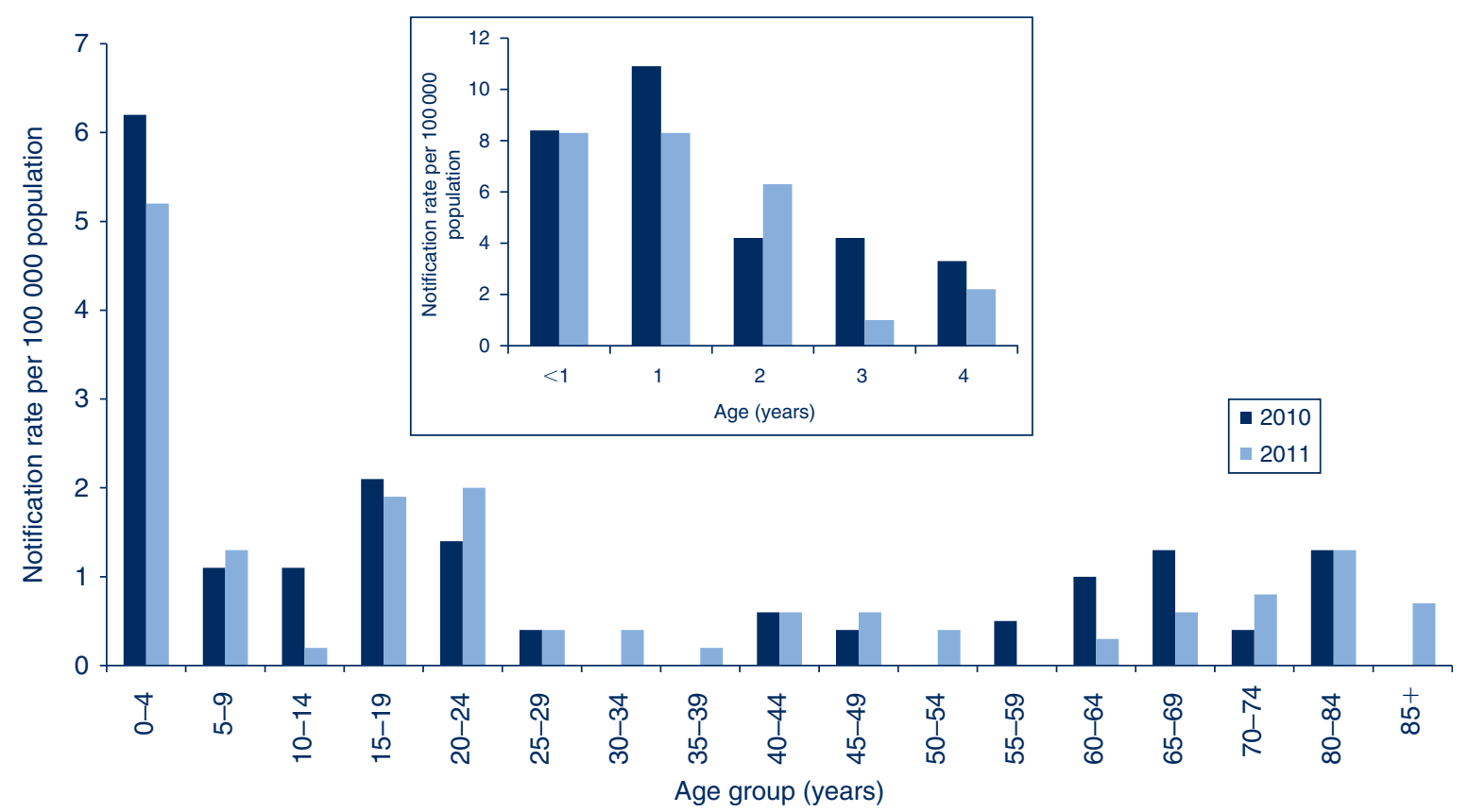

Figure 2. Comparison of annual case notification rates per 100000 population for invasive meningococcal disease by 5 -year age groups, NSW, 2010 and 2011. Comparison of annual case notification rates per 100000 population for children aged less than 5 years for each year of age, NSW, 2010 and 2011, presented inset. Source: NSW Notifiable Conditions Information Management System, Health Protection NSW.

were reported from children aged 13-24 months (eight cases, 8.3 per 100000 population) and infants aged less than 12 months (eight cases, 8.3 per 100000 population).

In 2011, 36 case-patients (50\%) with invasive meningococcal disease were male. Seven case-patients were Aboriginal people, amongst whom notification rates were 4.6 times higher $(95 \% \mathrm{CI} 2.1,10.0)$ than in non-Aboriginal people (4.2 and 0.9 per 100000 , respectively) (Figure 1). Geographically in NSW, the highest case notification rates were from Western NSW LHD (2.2 per 100000 population).

Of the 72 cases notified in NSW in 2011, serogroup information was recorded for 54 (75\%). Of these 54 case-patients, for $44(81 \%)$ disease was caused by serogroup B infection (for which there is no vaccine); $32 \%$ of these case-patients were aged less than 5 years, $57 \%$ were $>5$ years and $<65$ years of age, and $11 \%$ were $>65$ years of age. For four case-patients (7\%), disease was caused by serogroup Y infection; of these people three were aged less than 25 years of age and one was aged between 25 and 80 years. For four case-patients (7\%), disease was caused by serogroup W135 infection (of these people three were less than 2 years of age and one was aged between 2 and 40 years). Only two case-patients (4\%) had disease caused by serogroup $\mathrm{C}$ infection, one aged 32 years, the other 69 years - both were ineligible for vaccination and were not vaccinated. Of the 18 cases $(25 \%)$ of unknown serogroup, two were unable to be typed and eight had clinical findings consistent with meningococcal disease but no laboratory confirmation. Of the seven case-patients who were Aboriginal people, for four disease was due to serogroup B infection, two W135 infection and one due to serogroup Y. Information describing vaccination status was complete for 54 case-patients (75\%); there were no cases caused by serogroup $\mathrm{C}$ among people who had been vaccinated against serogroup $\mathrm{C}$.

\section{Mumps}

In 2011, 65 cases of mumps were notified in NSW compared to 39 in 2010 . The highest case notification rates of mumps were among young adults aged 20-24 years at onset of illness ( 11 cases, 2.2 per 100000 population). In 2011, 35 case-patients (54\%) were male.

In NSW, notified cases of mumps are not routinely followed up by public health units. No outbreaks of mumps were reported in 2011.

\section{Pertussis}

In 2011, 13053 cases of pertussis were notified in NSW (the highest level on record), compared with 9332 in 2010. The highest age-specific pertussis case notification rates were in children aged 5-9 years (4120 cases, 923.1 per 100000 population) and $10-14$ years (2334 cases, 514.7 per 100000 population), an increase from 2010 when 2744 cases (620.0 per 100000 population) and 1627 cases (359.4 per 100000 population) were notified in the 5-9 and 10-14-year age groups respectively (Figure 3 ). The greatest increase from 2010 to 2011 was among children aged 6 years $(958.0$ per 100000 population in 2011 


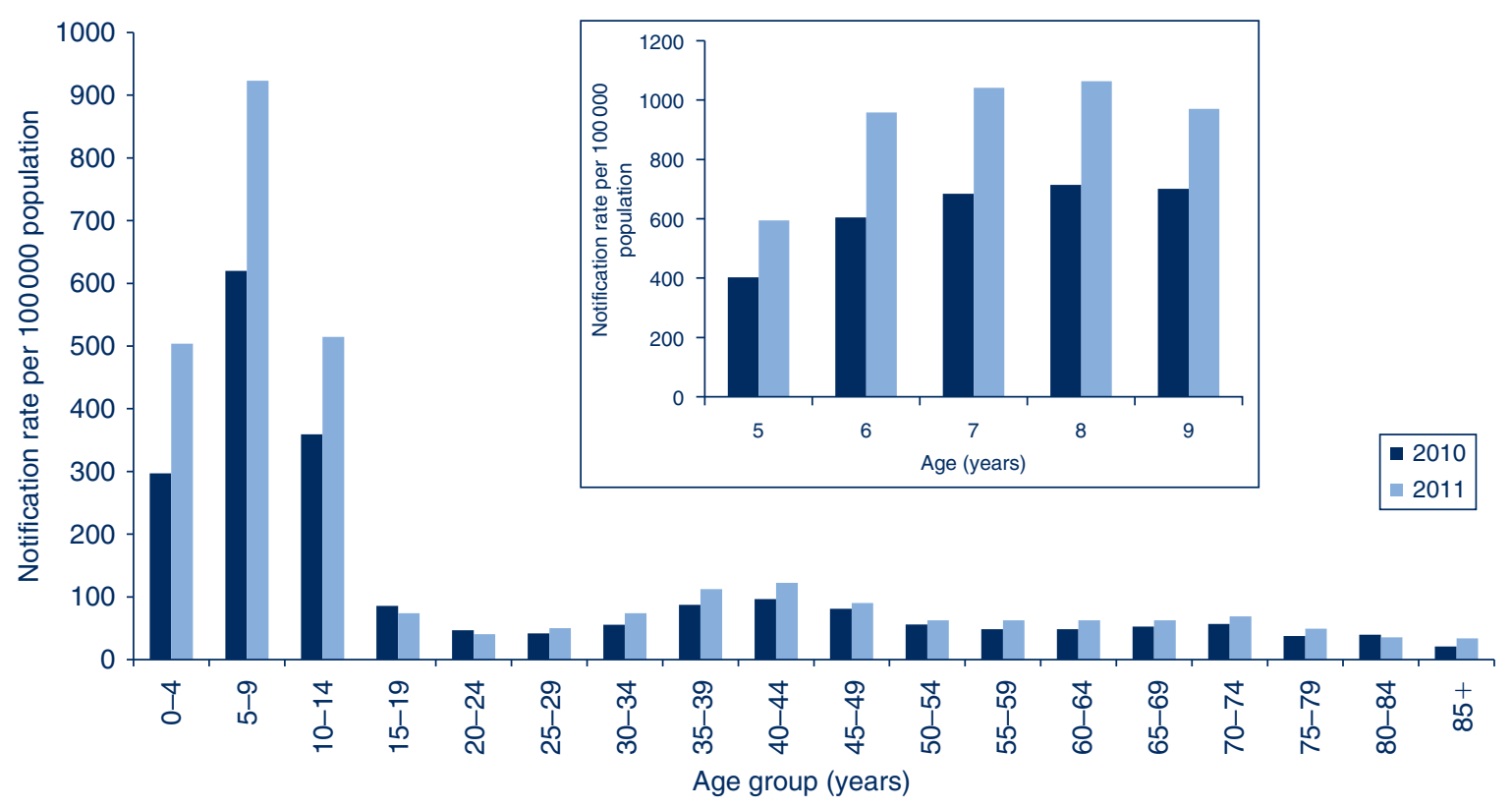

Figure 3. Comparison of annual case notification rates per 100000 population for pertussis by 5 -year age groups, NSW, 2010 and 2011. Annual case notification rates for children aged 5-9 years for each year of age, NSW, 2009 and 2010, presented inset.

Source: NSW Notifiable Conditions Information Management System, Health Protection NSW.

compared to 604.0 per 100000 population in 2010) and 7 years ( 1041.8 per 100000 population in 2011 compared to 683.9 per 100000 population in 2010). Of the casepatients aged less than 5 years, the highest notification rates were in children aged 3 years ( 588 cases, 601.8 per 100000 population) and 2 years (529 cases, 554.1 per 100000 population). One death was reported in a 1-month old infant, who was too young to be vaccinated.

In 2011, 5939 case-patients (45\%) were male. Of the 2403 case-patients aged $0-4$ years (who are followed up by public health units), 132 (5\%) were Aboriginal children. Geographically, the highest case notification rates were reported in Murrumbidgee (including Albury) (440.2.0 per 100000 population) and Nepean Blue Mountains (332.7 per 100000 population) LHDs.

In total, 520 case-patients were aged less than 12 months. Of these, $309(59 \%)$ were infants too young to have received three doses of vaccine (i.e. aged 6 months or less at onset of illness). Of the 1885 case-patients who were children aged 1-4 years, $247(7 \%)$ were reported to be not immunised, $31(2 \%)$ reported less than three doses of vaccine, and $1618(86 \%)$ reported three or more doses. For the remainder of case-patients $(5 \%)$ data on vaccine doses was not reported.

\section{Pneumococcal disease (invasive)}

In 2011, 524 cases of invasive pneumococcal disease were notified compared to 500 in 2010. Fifty-seven deaths were identified in 2011: one case-patient who died was a child aged less than 2 years who was fully vaccinated (due to disease caused by a serotype that was not included in the vaccine), nine case-patients who died were aged between 80 and 84 years and 15 were aged 85 years and over.

The highest case notification rates of invasive pneumococcal disease were in adults aged older than 85 years (55 cases, 37.9 per 100000 population) and 80-85 years (40 cases, 26.3 per 100000 population), and in children aged less than 5 years (70 cases, 14.7 per 100000 population) (Table 1). Of the case-patients less than 5 years of age, the highest notification rates were in infants aged less than 12 months (21 cases, 21.8 per 100000 population) and children aged 12-23 months (20 cases, 20.8 per 100000 population).

Fifty-two percent of case-patients were male. Of the 383 case-patients aged $0-4$ years or older than 50 years (who are followed up by public health units), 12 (3\%) were Aboriginal people. Geographically, the highest notification rates were reported in Western NSW (41 cases, 15.3 per 100000 population), Nepean Blue Mountains (36 cases, 10.4 per 100000 population) and Illawarra Shoalhaven (35 cases, 9.0 per 100000 population) LHDs (Table 2).

From 1 July 2011, 13-valent conjugate pneumococcal vaccine (PCV-13) replaced 7-valent conjugate pneumococcal vaccine (PCV-7) on the NSW Immunisation Program. The PCV-13 vaccine includes the additional serotypes 1, 3, 5, 6A, 7F and 19A.

\section{Rubella}

In 2011, 17 cases of rubella were notified in NSW compared to 13 in 2010. All case-patients were people aged between 15 and 60 years. Seven case-patients (41\%) 
were male. Geographically, the highest notification rates were in the Sydney LHD (0.5 per 100000 population). Notifications have not changed over the previous 5 years. There were no case notifications of congenital rubella.

\section{Tetanus}

In 2011, one case of tetanus was notified in NSW. This case-patient resided in Sydney LHD and was an 18-year old male who reported being vaccinated 12 years ago.

The number of notified cases of tetanus has remained relatively stable over the past 5 years, ranging from 1 to 2 cases annually. Most tetanus cases occur in older adults who are not adequately immunised. This was the youngest case-patient notified in the last decade in NSW.

\section{Discussion}

While there are limitations to the data, ${ }^{1}$ vaccinepreventable disease surveillance in NSW enables the implementation of timely public health measures, permits a better understanding of disease trends and helps inform policy. Notifications of some vaccine-preventable diseases (such as $H$. influenzae serotype b, invasive meningococcal disease, pneumococcal disease, mumps and rubella) have remained stable or declined over recent years.

Outbreaks of measles continue to occur, mostly as a result of people who are unvaccinated or have incomplete vaccinations travelling to countries where measles transmission is common and then returning home whilst infectious or during their incubation period. As measles is a highly infectious disease, local transmission can occur among the susceptible members of the contact network of these travellers, including infants too young to be protected through vaccination. In addition, there were 67 cases (including 11 clusters affecting 32 people) with no direct links to overseas travel. These cases were therefore thought to be locally acquired from infectious measles patients that may not have been diagnosed or sought medical attention. This highlights the challenges to comprehensive identification and notification of cases of measles where many clinicians may not have previously seen or diagnosed measles.

Pertussis remains an important public health problem in NSW. Public health activities should focus on the prevention of severe disease (which frequently occurs in young infants) and death (which mostly occurs in infants less than 2 months of age). One important recommendation (according to the Australian Immunisation Handbook) is that new parents, grandparents and adult carers of infants are vaccinated. ${ }^{6}$ While vaccine-induced selection pressure on pertussis strains has been suggested as a possible explanation for the significant increases in notifications in Australia in recent years, ${ }^{7}$ this is unconfirmed. The greater contribution is likely to have been increased (and more sensitive) testing for pertussis. ${ }^{8}$ Vaccination remains the key prevention and control tool, especially in the prevention of severe pertussis and death.

The number of notified cases of invasive meningococcal disease has declined significantly since the National Meningococcal C Immunisation Program commenced in 2003. The greatest reduction in notified cases of meningococcal disease has been for serogroup $\mathrm{C}$, from 45 cases ( $29 \%$ of those with known serogroup) in 2003, to less than 10 cases annually over the last 5 years, and two cases (4\% of those with known serogroup) in 2011. The number of cases of meningococcal disease associated with serogroup B has also decreased over time, but remains the most commonly identified serotype. The notifications of other serogroups (W135 and Y) have remained relatively stable over time.

Vaccine-related invasive pneumococcal disease (IPD) has diminished since the introduction of the 7-valent conjugate pneumococcal vaccine (PCV-7) in 2005, whilst serotypes not covered by the PCV-7 have been steadily increasing. Serotypes 1, 3, 6A, 7F and 19A (included in the 13-valent pneumococcal vaccine (PCV-13)) have been responsible for up to $59 \%$ (predominantly serotype 19A: $36 \%$ ) of IPD in children aged less than 5 years since 2005. Following the introduction of PCV-13 in 2011, further reduction in IPD is expected in children. It remains to be seen whether similar reductions in IPD due to serotypes covered by PCV-13 in people aged $>5$ years will be equivalent to those seen with PCV-7.

\section{Conclusion}

This review demonstrates the value in ongoing systematic collection of vaccine-preventable disease data to highlight the challenges as well as significant improvements in disease control over time. While vaccine-preventable diseases remain generally well controlled in NSW, challenges remain to ensure ongoing population health protection. High vaccination coverage and timely vaccination for infants and children is important to maintain low rates of disease. Improving vaccination coverage among people from culturally and linguistically diverse communities as well as Aboriginal communities is crucial for successful disease prevention strategies. In addition, individuals planning international travel who are susceptible to measles or other vaccine-preventable diseases should be encouraged to receive relevant vaccinations prior to their departure. As cases of some diseases like measles have become rare, it is increasingly important to raise awareness among clinicians, and those training to be clinicians, of the diagnostic features and to have routine infection control practices in place to minimise transmission in health care settings. 


\section{Acknowledgments}

The authors would like to thank and acknowledge the NSW public health network including laboratory staff for their work in identifying and managing cases of vaccine-preventable disease in NSW.

\section{References}

1. Spokes PJ, Gilmour RE. NSW Annual Vaccine-Preventable Disease Report, 2010. NS W Public Health Bull 2011; 22(9-10): 171-8. doi:10.1071/NB11028

2. Department of Health and Ageing. Australian national notifiable diseases and case definitions. Australian Government Department of Health and Ageing. Available at: http://www.health. gov.au/casedefinitions (Cited 8 May 2012).

3. Jayamaha J, Binns PL, Fennell M, Ferson MJ, Newton P, Tran T et al. Laboratory diagnosis, molecular characteristics, epidemiological and clinical features of an outbreak of measles in a low incidence population in Australia. J Clin Virol 2012; 54(2): 168-73. doi:10.1016/j.jcv.2012.02.025

4. Hope K, Boyd R, Conaty S, Maywood P. Measles transmission in health care waiting rooms: implications for public health response. Western Pacific Surveillance and Response Journal (in press).
5. Measles virus nomenclature update: 2012. Wkly Epidemiol Rec 2012; 87(9): 73-81.

6. National Health and Medical Research Council. The Australian Immunisation Handbook. 9th ed. Canberra: Australian Government Department of Health and Ageing; 2008.

7. Octavia S, Sintchenko V, Gilbert GL, Lawrence A, Keil AD, Hogg G et al. Newly Emerging Clones of Bordetella pertussis Carrying prn 2 and ptxP3 Alleles Implicated in Australian Pertussis Epidemic in 2008-2010. J Infect Dis 2012; 205(8): 1220-4. doi:10.1093/infdis/jis 178

8. Kaczmarek MC, Lambert SB, Kelly HA, Ware R, Valenti L, Britt H. Seven-fold rise in likelihood of pertussis-test requests during Australian GP encounters, 2011-2011. Seven-fold rise in likelihood of pertussis-test requests during Australian GP encounters, 2011-2011. Darwin, NT, Australia: Public Health Association of Australia; 2012. p. 37.

*The Public Health Act 2010 (NSW) (http://www.health.nsw.gov.au/phact/)

The Public Health Act 2010 (NSW) was passed by the NSW Parliament in December 2010 and commenced on 1 September 2012. The Public Health Regulation 2012 was approved in July 2012 and commenced, along with the Public Health Act 2010 (NSW), on 1 September 2012. The objectives of the Regulation are to support the smooth operation of the Act. The Act carries over many of the provisions of the Public Health Act 1991 (NSW) while also including a range of new provisions. 